\title{
¿Hay dos presidentes en Argentina? Un análisis \\ COMPARATIVO DEL APOYO LEGISLATIVO EN LAS POLÍTICAS EXTERIOR Y DOMÉSTICA (2001-2014)*
}

\author{
Are there two presidents in Argentina? A comparative analysis of \\ legislative support for foreign and domestic policy (2001-2014)
}

\section{PEDRO FELIÚ RIBEIRO}

Universidad de São Paulo

\section{FRANCISCO URDINEZ}

Pontificia Universidad Católica de Chile

\begin{abstract}
RESUMEN
El presente trabajo prueba la tesis clásica de la Ciencia Política norteamericana sobre la existencia de dos presidentes en iniciativas legislativas. Analizando las votaciones nominales argentinas recientes, confirmamos la pertinencia de la tesis para sistemas presidencialistas y marcadamente multipartidarios. Luego, comparando las temáticas internacionales y domésticas, analizamos qué factores afectan el apoyo legislativo recibido por el Presidente. Encontramos que la aprobación popular está positivamente asociada al nivel de apoyo que el Presidente recibe en iniciativas domésticas y no en las internacionales, mientras que el uso de Decretos de Necesidad y Urgencia, el andar de la economía, la polarización ideológica entre los partidos en el Congreso y la Cámara en la que la iniciativa es presentada, son variables que afectan el apoyo legislativo.
\end{abstract}

Palabras clave: apoyo legislativo al presidente, política exterior, política doméstica, congreso argentino

\begin{abstract}
This paper makes two contributions to the classical thesis in American Political Science on the existence of "two presidents", as applied to the Argentine case. Analyzing rollcall votes for the recent period, we confirm the validity of the thesis in a markedly presidential and multiparty system. Furthermore, comparing international and domestic legislative top$i c s$, we analyze which factors affect the legislative support received by the president. Using robust regressions, we find that popular approval is positively associated with the level of support that the president received for domestic but not international initiatives. The use
\end{abstract}

\footnotetext{
Los autores agradecen al Consejo Nacional de Investigación de Brasil (CNPq) por el auxilio financiero bajo el proyecto 479342/2013-0 y a la Fundación de Apoyo a la Investigación Científica del Estado de São Paulo (FAPESP), proceso número 2014/03831-3. Este trabajo fue presentado y discutido en el seno del Centro de Estudios de Negociaciones Internacionales de la Universidad de São Paulo y los autores agradecen los comentarios recibidos por sus colegas en dicha reuniones. También agradecen las sugerencias hechas por los revisores anónimos. Todos los errores son responsabilidad absoluta de los autores.
} 
of Necessity and Urgency Decrees, the economy's performance, the ideological polarization between the parties in Congress, and the chamber in which the initiative is submitted are all variables that affect legislative support.

Key Words: presidential support in Congress, foreign policy, domestic policy, Argentina

\section{INTRODUCCIÓN}

¿Es igualmente difícil para un Presidente recibir apoyo en sus agendas de política externa y doméstica en el Congreso Nacional? De haber diferencias, ¿qué factores están asociados a la variación en los niveles de apoyo a las mismas? Ambas preguntas conforman una amplia línea de investigación sobre la influencia presidencial en las diferentes temáticas tratadas en el Congreso.

Según Eshbaugh-Soha (2010), el tipo de política puede afectar de manera significativa el apoyo legislativo del Presidente, es decir, dependiendo del tema tratado en el Congreso, el Presidente puede tener mayor o menor dificultad en promover su agenda. Dentro de las distinciones temáticas más exploradas en la literatura se encuentra la de la política doméstica comparada a la internacional (Milner y Tingley 2012). En el caso de los regímenes presidencialistas y multipartidarios, abundantes en América Latina, el éxito presidencial en el legislativo, desagregado por tipo de política 『sea doméstica o internacionales aún una agenda incipiente que, por su menor madurez, merece mayor investigación.

El análisis de los procesos legislativos, específicamente sobre la aprobación de las propuestas legislativas, ayuda a comprender la distribución del poder político en los regímenes democráticos, así también a evaluar el desempeño de las instituciones democráticas. De esa manera, quienes estudian sistemas presidencialistas, consideran al apoyo legislativo al Presidente como uno de los factores más relevantes de la estabilidad institucional de un régimen democrático (Shugart y Carey 1992). En el caso de América Latina, la cuestión es particularmente relevante en la medida que la literatura destaca la intromisión de un poder sobre el otro como un factor determinante en el quiebre de los regímenes democráticos de la región (McCoy 1971; Santos 1986; González y Gillespie 1994).

El trabajo hace un aporte a la línea de investigación centrada en el análisis del poder presidencialista en América Latina, a través de un estudio cuantitativo de los factores asociados al apoyo legislativo del Presidente en Argentina, desagregando por temáticas doméstica e internacional. El trabajo se centra en los períodos legislativos entre 2001 y 2014, contemplando todas las votaciones nominales ocurridas en el pleno del Senado y de la Cámara de Diputados en esos años. Por medio de análisis econométricos, estimamos el apoyo legislativo al Presidente en cada una de las temáticas abordadas, así como diversos factores políticos e institucionales ya considerados en la literatura. 
Los resultados indican que, para las decisiones en el plenario, las iniciativas presidenciales sobre política externa recibieron mayor apoyo legislativo que las domésticas. A su vez, la aprobación popular al gobierno, el tamaño de la coalición de gobierno en el legislativo, el uso de Decretos de Necesidad y Urgencia y el andar de la economía, son variables asociadas al mayor o menor apoyo que una propuesta presidencial irá a recibir.

El artículo está estructurado en cinco secciones. En el apartado subsecuente se revisan los principales argumentos causales presentes en la literatura sobre la distinción entre política doméstica y política exterior respecto del apoyo legislativo al presidente, así como las variables primordiales asociadas al desempeño legislativo presidencial en Latinoamérica, independiente del tema tramitado. En la tercera sección se detalla el diseño de la investigación, describiendo el método utilizado, las variables que componen el modelo y los efectos esperados según la teoría existente. En el cuarto capítulo se presentan los resultados empíricos, los que son discutidos por hipótesis y comparados a las iniciativas del Poder Legislativo para el mismo periodo. En la última parte, se concluye el trabajo y se discute su contribución a la literatura existente.

\section{REVISIÓN DE LA LITERATURA Y DEFINICIÓN DE LAS HIPÓTESIS}

La presente revisión de literatura está estructurada en dos subsecciones. La primera examina las especificidades del apoyo legislativo del Presidente estadounidense en temas de política externa y política doméstica. En esta literatura, se consideran las especificidades de la política externa, lo que contribuirá en la estimación de un modelo econométrico que será definido más adelante. La segunda subsección explora la literatura cuyo foco es el apoyo legislativo de los presidentes latinoamericanos. A pesar de que ésta bibliografía es reducida, contribuye a aportar hipótesis referentes a las especificidades de los sistemas políticos latinoamericanos.

Apoyo al Presidente en las arenas internacional y doméstica

Existe un debate aún abierto en la literatura en relación a cuál es la especificidad del comportamiento legislativo en materia de política externa. Por ejemplo, se argumenta que la anarquía del sistema internacional incentiva a los partidos políticos a comportarse de manera unificada en asuntos de política externa, apoyando los compromisos asumidos por el Poder Ejecutivo en la arena internacional de una manera no observada para cuestiones domésticas (Bjereld y Demker 2000). Así, mientras la arena doméstica se caracterizaría por una polarización partidaria y por disputas políticas, la internacional sería caracterizada por el consenso y la baja polarización partidaria (King 1986).

Algunos estudios han contestado a esta afirmación, argumentando que se observan similares niveles de aprobación en ambas arenas (McCormick et al. 
1997). Según esta visión alternativa, la política externa es semejante a cualquier otra política pública, y el comportamiento del legislativo no se diferencia en nada cuando se compara la política doméstica. El país y periodo estudiado parecen tener gran influencia en los resultados obtenidos.

El estudio del voto legislativo y del éxito del Presidente en temas de política externa posee una larga tradición en la literatura anglosajona de Ciencia Política (Dahl 1950; Edwards 1989; Lindsay y Ripley 1992; Meernik 1993; Conley 1999; Baldwin y Magee 2000; Xie 2004). Esta conforma un debate en el que se pueden observar dos perspectivas contrapuestas: la perspectiva del bipartidismo junto a la tesis de los "dos presidentes", por un lado, cuyo argumento afirma que la lucha política se "detiene" en la frontera nacional; y la perspectiva política, por el otro, cuyo argumento es que la política externa está sujeta a la misma disputa partidaria e ideológica que caracteriza al proceso decisorio doméstico (McCormick y Wittkopf 1990: 1077).

Sobre la tesis de los "dos presidentes", Wildavsky (1966) argumenta que los presidentes consiguen un mayor éxito en el ámbito internacional debido a que poseen ventajas constitucionales e institucionales en relación al Congreso Nacional en la temática. El Poder Legislativo acaba por delegar sus facultades al Ejecutivo, ya que éste cuenta con una burocracia especializada y con acceso al control privilegiado de información, lo que acaba dando la imagen de un comportamiento unificado entre ambos poderes en asuntos internacionales (Ripley y Lindsay 1993).

El oponerse al Presidente en asuntos de política externa puede, también, ser perjudicial para los líderes partidarios \en particular冈 y para los legisladores

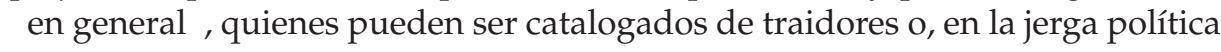
latinoamericana, de "vende patria". Por ende, hay un incentivo a evitar el conflicto con el Ejecutivo (Sinclair 1993). En el caso de presidencialismos latinoamericanos, hay ejemplos que vale la pena tocar. Alemán y Navia (2009: 413) demuestran que los tratados internacionales en Chile poseen mayores chances de aprobación en el Congreso comparados a proyectos legislativos de política doméstica y fiscal. Sanchez (2014), por su parte, analiza el caso de votaciones sobre Islas Malvinas en Argentina, un tema crítico para la política externa del país, llegando a la conclusión de que el éxito de las iniciativas del Poder Ejecutivo es mucho mayor al de las del Congreso, al tiempo que el número de iniciativas está correlacionada positivamente con su tasa de éxito, lo que corrobora la idea de que los presidentes, cuando dan inicio a un proyecto de ley, evalúan anticipadamente las posibilidades que tiene su aprobación.

Por otro lado, contrariando a los estudios que afirman la existencia de "dos presidentes" en la formulación de políticas en Estados Unidos (Holsti y Rosenau 1986; Canes-Wrone et al. 2008), algunos argumentan que el fin de la Guerra Fría ha producido un significativo descenso en el grado de unidad de los partidos Demócrata y Republicano en política externa, disminuyendo así el éxito presidencial en dicha temática a partir de 1990 (Kupchan y Trubowitz 
2007). Nuevamente se hace evidente que estos estudios son altamente sensibles al contexto histórico que se estudie.

Al contrario de una delegación del poder desde los partidos hacia el Poder Ejecutivo, la política externa norteamericana se caracterizaría por una creciente polarización en el plenario (Fleisher et al. 2000). Factores domésticos 『como las disputas ideológicas en el congreso (McCormick y Wittkopf 1992), las características económicas de los distritos electorales (Hiscox 2002; Heinz y Mansfield 2006), la influencia de intereses organizados (Fordham 1998) y de la opinión pública (Burgin 1993) $\bigotimes$ desempeñan un papel importante en la determinación de los votos de los legisladores en el proceso decisorio de la política externa de los Estados Unidos.

Frente a la problemática expuesta, elaboramos la primera hipótesis a ser probada para el caso argentino reciente:

$H_{1}$ : El Presidente argentino recibe mayor apoyo legislativo en sus iniciativas referentes a política exterior que en las de política doméstica.

Además de la variación en el apoyo legislativo según el tema a tratar (internacional o doméstico), también creemos que puede haber una variación en relación a los subtemas de política externa. Por ejemplo, Prins y Marshall (2001) argumentan que la distinción de asuntos de defensa nacional y de seguridad internacional (temas de high politics) y los demás tópicos de política externa, como la política comercial (temas de low politics), tiene un efecto sobre el apoyo legislativo del Presidente. El argumento utilizado es que asuntos de seguridad exterior ganarían mayor consenso en las cámaras. Utilizamos como control en nuestro análisis la temática de política exterior tramitada en pleno del Congreso Nacional de Argentina. A continuación repasamos los principales determinantes del apoyo presidencial en el legislativo, específicos para presidentes de América Latina, de acuerdo a la literatura preexistente.

\section{Determinantes del apoyo legislativo a los presidentes latinoamericanos}

Uno de los mayores debates en torno a la redemocratización de América Latina a fines de los años 80 del siglo pasado giraba en torno a los niveles de estabilidad institucional de las nuevas arquitecturas institucionales que emergían luego de acabadas las dictaduras. Parte de la literatura especializada juzgaba que la combinación entre multipartidismo y sistemas presidencialistas era propensa a la inestabilidad, amenazando la supervivencia de los nuevos regímenes democráticos en la región (Mainwaring 1993; Stepan y Skach 1993).

Incluso cuando estas previsiones pesimistas no se hayan materializado (Pereira y Melo 2012), actualmente existen conflictos políticos entre los poderes Ejecutivo y Legislativo que siguen siendo centrales en las agendas de investigación sobre regímenes presidencialistas (Alemán y Navia 2009: 401). Los trabajos sobre éxito 
presidencial en América Latina han buscado combinar la prueba de hipótesis presentes en la literatura del presidencialismo norteamericano con aspectos institucionales específicos a los sistemas presidencialistas latinoamericanos, particularmente el multipartidismo.

Un primer aspecto relevante para la comprensión del éxito del Presidente, referido al contexto político, es el tamaño de su base de apoyo en el Legislativo, ya que los mandatarios cuyo partido o coalición de partidos fuese minoritaria tendrían grandes dificultades en aprobar sus agendas de gobierno (Alcántara y Montero 2008; Figueiredo et al. 2009). Diferente del caso estadounidense, cuya medida de mayoría legislativa se restringe al partido político del Presidente, son los presidencialismos multipartidarios, en los que es común la formación de coaliciones partidarias (Chasquetti 2001; Cheibub et al. 2004; Negretto 2006).

Por esta razón es importante contabilizar no solo el porcentaje de asientos que el partido del Presidente posee, sino también la cantidad de asientos correspondientes a la coalición como un todo. Para el caso argentino, Alemán y Calvo (2008) no hallaron una relación estadísticamente significativa entre el nivel de apoyo presidencial en el Congreso y su éxito legislativo durante el periodo 1983-2001. Formulamos la misma hipótesis que los autores mencionados (Alemán y Calvo 2008: 13) con el objetivo de probarla en un período más reciente al de ellos y con votaciones nominales (de 2001 a 2014):

$\mathrm{H}_{2}$ : El tamaño de la coalición legislativa del Presidente está positivamente relacionado al nivel de apoyo legislativo del mismo.

En lo que respecta al apoyo legislativo del Presidente, hay algunas variables relevantes en la literatura que deben ser consideradas como controles en nuestro modelo de regresión. La primera de ellas es el número efectivo de partidos (NEP). De manera general, cuanto mayor el NEP, más difícil se hace la formación de coaliciones mayoritarias y mayor será la probabilidad de polarización ideológica, dificultando la aprobación de las iniciativas legislativas del Presidente (Cox y McCubbins 2001; Garcia Montero 2009).

En esta dirección, Santos et al. (2014) sostienen que, en el caso latinoamericano, la elevada polarización ideológica entre los partidos políticos en el Congreso conduce al Presidente a la pérdida de control sobre la agenda legislativa $\mathrm{y}$, por ende, a ver su apoyo legislativo perjudicado. Por ello, creemos que es importante tener en cuenta en el análisis, variables como el número efectivo de partidos (NEP) y la polarización ideológica entre los principales partidos de las legislaturas analizadas.

A su vez, la utilización de Decretos de Necesidad y Urgencia (DNU) es apuntada en la literatura como elemento relevante en la comprensión del apoyo legislativo de los presidentes latinoamericanos, una vez que posibilitan a estos la coordinación de la agenda legislativa (Figueiredo y Limongi 1999; Alemán y Tsebelis 2005). Alemán y Calvo (2008) sostienen que el apoyo legislativo de los presidentes argentinos está negativamente asociado a la utilización de DNU, 
sugiriendo que presidentes impopulares acaban utilizando mucho este recurso para logar la aprobación de sus iniciativas legislativas. Así, formulamos la tercera hipótesis del trabajo:

$H_{3}$ : La utilización de DNU está negativamente asociada al nivel de apoyo legislativo que recibe el Presidente.

Hay dos elementos más en la literatura asociados al apoyo legislativo del Presidente, concernientes a las instituciones y contextos políticos. El primero es fruto del bicameralismo, o sea, de la distinción entre tramitación de las propuestas legislativas del Presidente en la Cámara de Diputados y en el Senado. Alemán y Calvo (2008) argumentan que las reglas bicamerales en Argentina ofrecen ventajas en relación a la casa legislativa en que la propuesta del Presidente es iniciada. Así, el Presidente tendría un incentivo para aprovechar estas ventajas e iniciar su propuesta legislativa en la casa que ofrezca mejor relación costobeneficio.

En segundo lugar, la literatura ha encontrado evidencias de que los presidentes recién elegidos gozan de mayor apoyo de los legisladores debido al efecto reciente de las elecciones, denominado "luna de miel" (honey-moon period, ver Mack et al. 2011). Hay ejemplos empíricos en los presidencialismos latinoamericanos del efecto de "luna de miel" en Uruguay (Altman 2000), Paraguay (Molinas et al. 2004), Colombia (Cárdenas et al. 2006) y Chile (Alemán y Navia 2009). Incluiremos como variable de control, entonces, a la casa legislativa en la que la propuesta presidencial fue analizada y controlaremos por el efecto de "luna de miel" en los meses iniciales de cada mandato.

Un aspecto central, que aún no hemos mencionado, es el nivel de apoyo popular que el Presidente recibe durante su mandato. La lógica es que presidentes con mayor apoyo popular, tendrán mayor apoyo de los legisladores al presentar sus iniciativas, una vez que rechazarlas pueda significar en un costo electoral para el legislador (Rudalevige 2002). La relación positiva entre aprobación popular y aprobación legislativa del Presidente ha sido hallada en el caso brasilero (Mainwaring 1997), uruguayo (Altman 2000) y argentino (Calvo 2007). No obstante, esta relación positiva no genera consenso en la literatura, habiendo algunos trabajos que muestran bajo o nulo efecto de la aprobación popular presidencial sobre su éxito legislativo (Bond y Fleischer 1990). Por ejemplo, para el caso chileno, Alemán y Navia (2009) no encontraron un efecto estadísticamente significativo en la asociación entre la popularidad del presidente chileno y su éxito legislativo. Formularemos la cuarta hipótesis de este trabajo, contribuyendo a este debate:

$H_{4}$ : la aprobación pública del Presidente está positivamente asociada al nivel de apoyo legislativo del mismo.

A continuación, y habiendo definido todas las hipótesis de este trabajo, detallamos el diseño de la investigación y la estrategia empírica. 


\section{DISEÑO DE INVESTIGACIÓN}

Nuestro universo observacional está compuesto por todas las votaciones nominales tramitadas en el pleno del Senado y Cámara de Diputados en Argentina entre 2001 y 2014, obtenidas por medio de la aplicación de visualización de votaciones nominales Década Votada (2001-2014), cuya fuente original de datos es el propio sitio web del Congreso Nacional. El período comprende cuatro presidentes y cinco mandatos, totalizando 3.377 votaciones, 1.921 en el Senado y 1.456 en la Cámara. Del total de votaciones, 2.965 fueron clasificadas como de política doméstica en cuanto 412 como de política exterior.

Las votaciones nominales fueron frecuentemente utilizadas como unidad de análisis en investigaciones sobre el éxito legislativo del Presidente en las temáticas doméstica e internacional (Schraufnagel y Shellman 2001; Mack et al. 2011). Como las votaciones nominales constituyen una muestra de todas las leyes aprobadas, es importante considerar las reglas internas de cada casa legislativa argentina para el registro nominal de las votaciones. En la Cámara, todas las votaciones en general son nominales (desde 2006), así como cualquier diputado puede solicitar el voto nominal mediante el apoyo de un décimo de los legisladores presentes (desde 1997). Así como en el Senado, todas las elecciones internas de la Cámara son nominales, pero en el caso del primero, todas las otras votaciones se registran nominalmente mediante solicitación de un senador con el apoyo de la mayoría absoluta del Senado. ${ }^{1}$

En ese marco institucional, como argumentan Molinelli et al. (1999), las muestras de votaciones nominales en el Congreso Nacional de Argentina contienen la mayor parte de las legislaciones más importantes allí tramitadas. También se espera que, para enmiendas controvertidas, sea solicitado el registro nominal. Sin embargo, como argumenta Calvo (2013), las votaciones nominales en la Cámara de Diputados son generalmente precedidas por un acuerdo entre los liderazgos con mayoría parlamentaria, lo que explicaría, en cierta forma, los altos índices de disciplina partidaria en este país. Siendo así, el consenso entre bloques es el fruto de un extenso trabajo previo al proyecto a ser discutido en el plenario.

Aquellos temas que pueden dividir los liderazgos dentro del partido mayoritario son mantenidos al margen del plenario. El control de la agenda legislativa, por parte de los liderazgos partidarios, vuelve difícil que un proyecto legislativo del ejecutivo sea rechazado por votación (Calvo 2013). Por este motivo, el análisis de las votaciones nominales captura mejor el nivel de polarización en el posicionamiento público de los legisladores argentinos, estructurados en bloques fuertemente disciplinados. 
Las votaciones nominales son generalmente requeridas cuando los líderes de los bloques opositores quieren que sus votos, y los del oficialismo, sean registrados públicamente (Jones y Hwang 2005). Así, las votaciones nominales también pueden ser importantes para los partidos políticos y legisladores, ya que tornan más visible al electorado sus posicionamientos en temas que pueden ser sensibles a sus pretensiones electorales. Como será observado a continuación, aunque hayan existido acuerdos previos a las votaciones, observamos que la diferenciación entre apoyo a materias de política externa y doméstica es marcadamente significativa. Dicho esto, pasamos a describir la instrumentalización de la variable dependiente de nuestro estudio.

\section{Variable dependiente}

Construimos nuestra variable dependiente utilizando la proporción de votos favorables en la votación de ambas casas legislativas, desconsiderando los legisladores ausentes (Mack et al. 2011). Es decir, para cada votación, calculamos el total de votos a favor sobre el total de votos presentes en cada cámara. El objetivo central es medir la magnitud del apoyo legislativo al Presidente más allá del rechazo o aprobación de las materias tramitadas en el Congreso Nacional. Buscamos capturar variaciones en el porcentaje de apoyo que recibe el Presidente en las distintas temáticas tramitadas.

\section{Variables independientes - Variables de interés central}

Presentamos abajo la instrumentalización de las variables independientes para probar las hipótesis presentadas en la sección anterior:

\section{Política exterior}

Las votaciones de política exterior, según la clasificación descrita anteriormente, fueron codificadas como " 1 ", mientras que las votaciones de política doméstica fueron codificadas como " 0 ". Se identifican como votaciones de política exterior aquellas que hacen referencia directa o poseen consecuencias conocidas en entidades extranjeras. Se incluyen en ese criterio, votaciones referentes a la ratificación de tratados y acuerdos internacionales firmados con países extranjeros y organizaciones internacionales, salvaguardias proteccionistas, reglamentación aduanera, subsidios a la exportación, modificaciones de leyes internas para adecuación de tratados internacionales, medidas conducentes al funcionamiento del Ministerio de Relaciones Exteriores y sus representaciones diplomáticas, solicitaciones al Poder Ejecutivo que aborden temas internacionales, acciones y reglamentaciones referentes a la defensa nacional. 


\section{Aprobación popular del Presidente}

Los datos de aprobación popular de los presidentes argentinos fueron obtenidos del Índice de Confianza en el Gobierno (ICG) de la Escuela de Gobierno de la Universidad Torcuato Di Tella para los años 2001 a 2014. Más específicamente, utilizamos la pregunta sobre aprobación o desaprobación del gobierno del Presidente, resultando en el porcentaje de personas que aprueban el gobierno en el período en que la materia es votada en el pleno del Congreso ${ }^{2}$.

\section{Tamaño de la coalición del gobierno}

El tamaño de la coalición del gobierno en la Cámara y Senado fue establecido por el porcentaje de sillas de los partidos políticos que, formalmente, declararan apoyo al Presidente. En el último año del gobierno de De la Rúa, la coalición entre UCR y FREPASO estaba deshecha y el presidente contaba con un apoyo minoritario (Sanchez 2015), siendo contabilizados solo los legisladores de UCR. En el gobierno de Duhalde, hubo una alianza entre el PJ, UCR y FREPASO (Kasta 2009), siendo contabilizados los legisladores de esos partidos para cada casa legislativa. En el caso del gobierno de Néstor Kirchner, los legisladores del PJ fueron considerados oficialistas.

Para el primer gobierno de Cristina Kirchner, aunque hubo disidencias en el PJ (Catterberg y Palanza 2012), la disciplina partidista del PJ se mantuvo elevada (Feliú y Onuki 2014), siendo también contabilizados todos los legisladores del PJ como oficialistas. Durante el segundo gobierno de Cristina Kirchner, particularmente a partir de 2013, la escisión del PJ se hizo evidente, por tanto distinguimos entre peronistas alineados y peronistas disidentes, incluyendo los bloques de Juan Manuel de la Sota en Córdoba, Adolfo Rodiguez Saá en San Luis y Sergio Massa en Buenos Aires.

\section{$D N U$}

Medimos la cantidad de DNU por ciclo legislativo (de enero a noviembre) por medio del sistema argentino de información jurídica (Infojus) 3 . Hemos recopilado la cantidad de DNU aprobados y publicados en el Diario Oficial por año.

\footnotetext{
El indicador está diseñado de forma de captar lo que los ciudadanos piensan respecto de aspectos esenciales del gobierno nacional, a partir de la estimación de cinco dimensiones: (1) la imagen o evaluación general del gobierno, (2) la percepción sobre si se gobierna pensando en el bien general o en el de sectores particulares, (3) la eficiencia en la administración del gasto público, (4) la honestidad de los miembros del gobierno y (5) la capacidad del gobierno para resolver los problemas del país. El índice se presenta en una escala que varía entre un mínimo de 0 y un máximo de 5 . Accedido desde http://www.utdt.edu/.

Disponible en http://www.infojus.gob.ar/ Último acceso: diciembre de 2015.
} 


\section{Variables independientes - Variables de control}

\section{Polarización ideológica}

Para medir la polarización ideológica de cada período legislativo de nuestra muestra, utilizamos la misma estrategia que Badillo (2007: 167). Así, el índice de polarización ideológica es la diferencia entre la media (auto-ubicación) del valor más extremo a la izquierda y el valor más extremo a la derecha, dividiendo el resultado por nueve. La auto-ubicación de los legisladores argentinos fue obtenida con datos del Proyecto Elites Parlamentarias en América Latina $(\text { PELA })^{4}$. Mientras más cercano a 0 el indicador anual, más baja es la polarización ideológica apuntada por el índice. Análogamente, mientras más cercano de 1, mayor la polarización ideológica.

\section{Confianza en la economía}

La confianza del ciudadano argentino en la economía fue obtenida por medio del Índice de Confianza del Consumidor del Centro de Investigación en Finanzas de la Universidad Torcuato di Tella. El marcador calcula el porcentaje de respuestas positivas en relación a la economía en una muestra nacional. La serie histórica es medida mensualmente, siendo utilizado el índice del mes correspondiente a la fecha de la votación en el pleno de ambas las casas.

\section{Número efectivo de partidos (NEP)}

El NEP es obtenido por $N=\frac{1}{\sum_{i=1}^{n} P_{i}^{2}}$, donde $\mathrm{N}$ es el número de partidos con al menos una silla, y $P_{i}^{2}$ es el cuadrado de la proporción de sillas de cada partido político de la legislatura (Laakso y Taagepera 1979). Mientras mayor el índice, mayor el número efectivo de partidos en la legislatura.

\section{Luna de miel}

El período correspondiente a la "luna de miel" del Presidente es poco debatido en la literatura (Mack et al. 2011), siendo utilizado tanto el primer semestre de mandato como el primer año. En ese estudio, seguimos la misma estrategia adoptada por Alemán y Calvo (2008) y codificamos como “ 1 " el primer año del mandato presidencial y "0" los demás años. Abajo, en la Tabla 1, presentamos los promedios de las variables independientes según el presidente de nuestra muestra.

4 Éste proyecto es desarrollado por el Área de Ciencia Política y de la Administración de la Universidad de Salamanca junto al Instituto de Iberoamérica de la misma universidad, disponible en http://campus.usal.es/ acpa/. Último acceso: diciembre de 2015. 
Tabla 1: Promedio anual de las variables por presidente

\begin{tabular}{|c|c|c|c|c|c|c|c|}
\hline & $\begin{array}{l}0 \\
\stackrel{0}{0} \\
0 \\
.0 \\
00 \\
0 \\
0 \\
0 \\
0 \\
0 \\
0\end{array}$ & 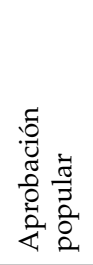 & $\stackrel{n}{\grave{n}}$ & 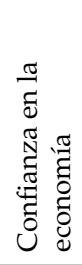 & 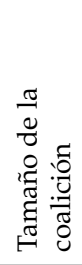 & 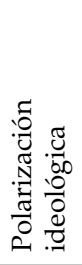 & 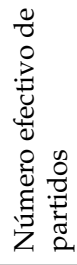 \\
\hline De la Rúa (2001) & 0,60 & 7,04 & 33 & 34,22 & 23,50 & 0,48 & 5,06 \\
\hline Duhalde (2002-2003) & 0,62 & 7,85 & 98,5 & 31,79 & 62,20 & 0,44 & 5,06 \\
\hline Néstor Kirchner (2003-2007) & 0,88 & 49,36 & 52,4 & 54,25 & 53,06 & 0,29 & 3,24 \\
\hline Cristina Kirchner (2007-2014) & 0,86 & 30,5 & 10,18 & 44,82 & 50,58 & 0,24 & 3,61 \\
\hline
\end{tabular}

Observamos cómo el apoyo legislativo fue marcadamente elevado durante todo el periodo estudiado, sobre todo durante el gobierno de los Kirchner. Los gobiernos de De la Rúa y Duhalde registran niveles bajísimos de aprobación popular, marcados por la histórica crisis económica de 2001, lo que también se refleja en los bajos valores de confianza en la economía.

El gobierno con mayor coalición de gobierno fue el de Duhalde, mientras que el de menor fue el de De la Rúa. El número efectivo de partidos pasó de cinco a poco más de tres a lo largo del periodo analizado. Finalmente, y propio al caso argentino, vale destacar que el número de DNU para los presidentes son frecuentes, pero con considerable variación entre los mandatarios. El caso más extremo es el de Duhalde, quien registra 99 DNU en su año de gobierno, seguido por Néstor Kirchner con promedio anual de 52,4, De la Rúa 33 y Cristina Kirchner 10,18.

\section{ESTRATEGIA EMPÍRICA Y RESULTADOS}

Muchos trabajos en la literatura revisada han utilizado modelos jerárquicos debido a la posibilidad que ofrecen para analizar los datos en clusters $^{5}$ (Alemán y Calvo 2008, 2010; Anderson y Singer 2008; Calvo y Sagarzazu 2011). La intención primera era rodar modelos jerárquicos de la misma manera que ellos. Sin embargo, la capacidad explicativa de los niveles del modelo resultaron ser insignificantes, debido, en gran parte, a la utilización de votaciones nominales $y$, por consecuencia, la fuerte concentración de la variable dependiente, como se aprecia mejor en la densidad acumulada de la misma en la Figura 1. 
Figura1: Densidad acumulada del apoyo legislativo

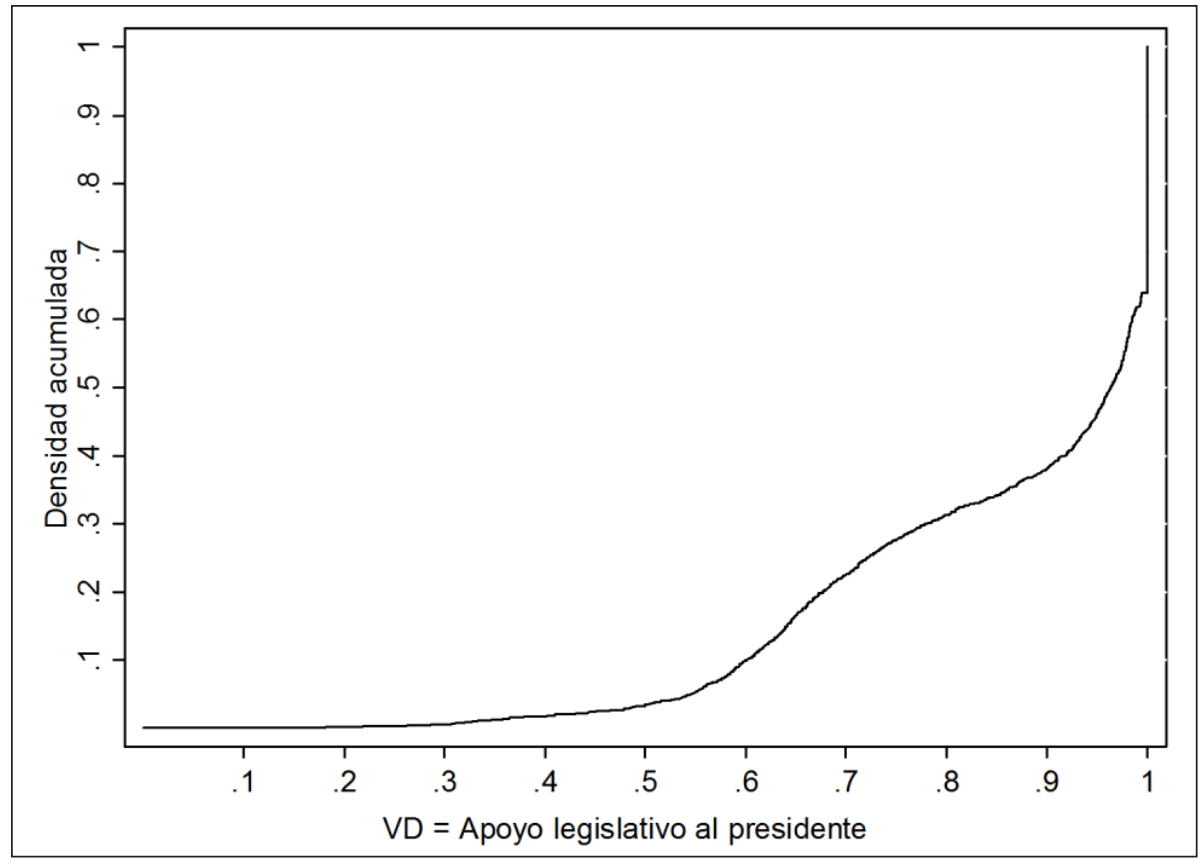

Una alternativa para lidiar con variables dependientes extremamente asimétricas es rodar regresiones cuartílicas. Este método resultó poco satisfactorio ya que solo conseguimos rodar regresiones para los tres primeros cuartiles, que es cuando la variable dependiente presenta su mayor variabilidad. Siendo que la literatura sobre análisis legislativa acostumbra analizar los diez cuartiles de la distribución (ver Bertelli y Richardson 2008; Teyssier 2011 y Häge 2013), la opción restante fue la de rodar regresiones robustas, método también utilizado en la literatura (Rousseeuw y Leroy 2005; Andersen 2008). ${ }^{6}$

La conclusión a la que llegamos fue que la polarización en la variable dependiente se debe a las características propias del proceso legislativo argentino, caracterizado por los elevados niveles de disciplina partidaria observados en las votaciones nominales. A pesar de esta limitación de los datos, es posible observar en la Figura 2 una distinción clara entre política exterior y política doméstica. Con excepción de 2014 en el gobierno de Cristina Kirchner, los proyectos presidenciales en política exterior votados en el plenario recibieron mayor apoyo que los proyectos de política doméstica. Adelante, analizamos con mayor precisión esa diferencia.

6 No confundir con el clásico método de regresión por Mínimos Cuadrados Ordinales con errores estándar robustos a la White (1980). 
Figura 2: Promedio anual del nivel de aprobación legislativa por presidente y tipo de política

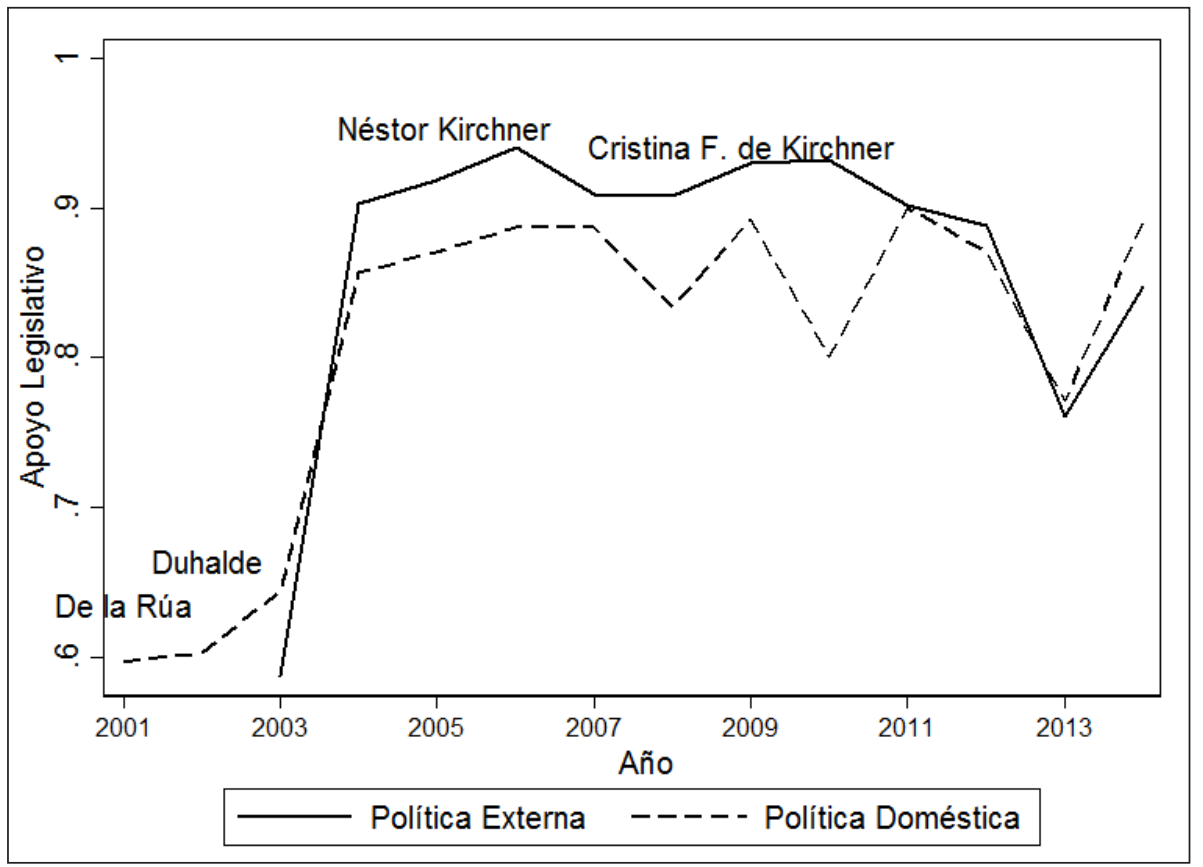

La principal justificativa para aplicar esta metodología es que, en el análisis de regresión linear, la presencia de valores atípicos puede distorsionar fuertemente el clásico estimador de mínimos cuadrados (MCO) y dar lugar a resultados poco fiables. El método de regresión robusta pondera de manera especial los valores atípicos para que su peso en la matriz de varianza-covarianza sea menor. ${ }^{7}$

Este modelo ha sido utilizado en trabajos de estudios legislativos, particularmente en los Estados Unidos (Radcliff 2001; Kuenzi y Lambright 2005) aunque es una técnica que no abunda en la literatura, sobre todo porque la distribución de la variable dependiente no suele ser un problema serio de los trabajos empíricos, lo que permite rodar regresiones por MCO. Vale aclarar que la lectura del efecto de las variables independientes sobre la dependiente es igual que en el modelo de $\mathrm{MCO}$, por lo que su elección no dificulta la interpretación de los resultados (Rousseeuw y Leroy 2005).

\footnotetext{
Aunque los coeficientes beta en una regresión pueden calcularse de varias maneras, la idea subyacente es siempre tratar de llegar lo más cerca posible del valor verdadero al reducir la magnitud de los residuos, medida por un error de predicción agregada. Un inconveniente del método por MCO es que, al considerar los residuos al cuadrado, tiende a conceder una importancia excesiva a las observaciones con grandes residuos y, en consecuencia, distorsionar la estimación de parámetros en el caso de la existencia de valores atípicos. El modelo de regresión robusta es bastante útil en escenarios como este y cuando no surtieron efecto transformaciones en la variable dependiente (transformaciones logarítmicas o de raíz cuadrada).
} 
El modelo de nuestro trabajo analiza las iniciativas legislativas del presidente en perspectiva comparada con las iniciativas del propio Poder Legislativo. Ha sido apuntado en la literatura, con razón, que generar inferencias estadísticas sobre el éxito legislativo del Presidente sin compararlo al éxito del Poder Legislativo es ver apenas una cara de la moneda (Alemán y Calvo 2010). Nuestra base de datos está compuesta por 1.094 votaciones nominales de iniciativas presidenciales (32\%) y 2.283 iniciativas del propio legislativo (68\%). Así, nuestro modelo de base es:

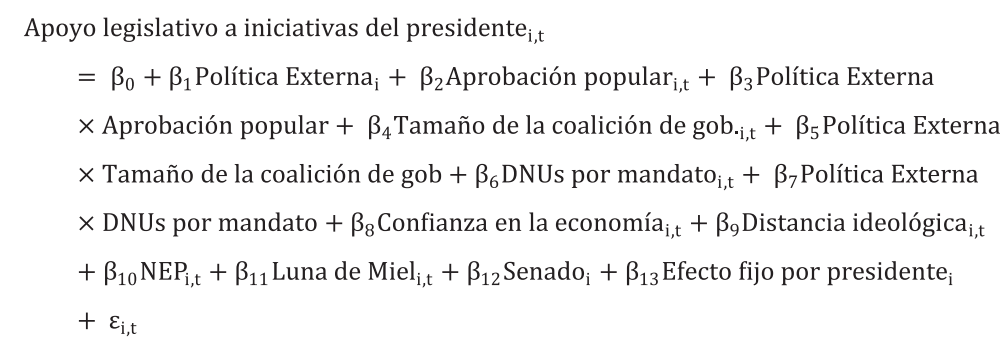

A su vez, estimaremos un modelo de comparación con iniciativas legislativas equivalente al modelo para iniciativas del Presidente, como se aprecia en la Tabla 2. En este último, las variables DNU, aprobación popular y "luna de miel" no fueron incluidas ya que están exclusivamente relacionadas a iniciativas presidenciales. Hemos recurrido a variables interactivas para probar cada una de nuestras hipótesis, comparando iniciativas de política doméstica e internacional. Como las variables interactivas suelen ser difíciles de interpretar para los lectores, hemos creado un gráfico de efectos marginales por cada modelo, para facilitar la lectura cuando estas variables tuvieron efectos estadísticamente significativos.

Tabla 2: Modelos de regresión robusta

\begin{tabular}{lcc}
\hline & $(1)$ & $(2)$ \\
\hline & $\begin{array}{c}\text { Iniciativas } \\
\text { Presidenciales }\end{array}$ & $\begin{array}{c}\text { Iniciativas } \\
\text { Legislativas }\end{array}$ \\
\hline Política Externa & $0.49^{* * *}$ & 0.016 \\
Aprobación popular & $(4.36)$ & $(0.86)$ \\
Política Externa $\times$ Aprobación popular & $0.0054^{* *}$ & - \\
Tamaño de la coalición de gobierno & $(3.25)$ & - \\
& $-0.0063^{* * *}$ & - \\
Política Externa $\times$ Tamaño de la coalición de gobierno & $(-5.08)$ & $0.0046^{* * *}$ \\
& 0.0038 & $(7.45)$ \\
\end{tabular}




\begin{tabular}{lcc} 
DNUs & $-0.013^{* *}$ & - \\
Política Externa $\times$ DNUs & $(2.99)$ & - \\
& $0.0031^{* * *}$ & - \\
Confianza en la economía & $(4.39)$ & -0.000097 \\
& $0.0057^{* *}$ & $(-0.06)$ \\
\hline Polarización ideológica & $(2.72)$ & $-6.13^{* * *}$ \\
& $-10.29^{* *}$ & $(-49.78)$ \\
NEP & $(-3.44)$ & $0.028^{* * *}$ \\
& 0.04 & $(5.61)$ \\
Luna de Miel & $(0.24)$ & - \\
& 0.009 & -
\end{tabular}

Interceptos por presidente (Cristina F. de Kirchner es referencia)

\begin{tabular}{lcc}
\hline De la Rúa & $2.78^{* *}$ & $1.09^{* * *}$ \\
& $(3.12)$ & $(31.27)$ \\
Eduardo Duhalde & $1.19^{* * *}$ & $0.51^{* * *}$ \\
& $(4.32)$ & $(38.91)$ \\
Nestor Kirchner & $0.63^{* *}$ & $0.34^{* * *}$ \\
& $(2.97)$ & $(58.41)$ \\
\hline Constante & 1.67 & $2.175^{* * *}$ \\
& $(1.77)$ & $(33.62)$ \\
$\mathrm{R}^{2}$ & 1094 & 2283 \\
\hline
\end{tabular}

Nota:Test-t entre paréntesis. Significancia estadística expresada como: ${ }^{*} \mathrm{p}<0.05,{ }^{* *} \mathrm{p}<0.01,{ }^{* * *}$ $\mathrm{p}<0.001$. Efectos fijos por año fueron calculados pero no reportados en la tabla.

Como podemos observar en la Figura 3, correspondiente al modelo 1 de la Tabla 2, cada curva posee un punto de origen propio y una inclinación de curva propia, lo que muestra que el apoyo a las iniciativas presidenciales es totalmente diferente si son de política doméstica o de política exterior. Comenzamos por nuestra primera hipótesis, referente a la tesis de los dos presidentes: Entre 2001 y 2014, el Presidente recibió mayor apoyo legislativo en sus iniciativas referentes a política exterior que en las de política doméstica. En otras palabras, este resultado demuestra que las negociaciones previas a la votación entre los bloques producen mayor consenso en asuntos de política exterior que doméstica. 
La aprobación de la agenda de política externa del Presidente en el legislativo posee una alta especificidad cuando se la compara a otros temas, ya que el Presidente posee la prerrogativa de negociar y firmar un acuerdo internacional (artículo 72 de la Constitución argentina). De esta manera, oponerse a una propuesta presidencial puede significar afectar la credibilidad de un país en el ámbito internacional, haciendo que un veto sea altamente costoso para el legislador. Junto a este motivo, la mayor capacidad institucional del Poder Ejecutivo en la conducción de asuntos externos incentiva al congreso a delegar esta materia. Finalmente, los temas internacionales tienden a generar un menor efecto distributivo en el ámbito doméstico, lo que los vuelve poco interesantes en el debate electoral.

Dentro de los subtemas de política externa tramitados en el Congreso argentino en nuestra muestra, los de política comercial son los que presentan mayor grado de polarización, justamente por ser los que poseen efectos distributivos más acentuados. A pesar de que la participación del Congreso argentino en política externa haya sido descrita como meramente delegativa (Sanchez 2015), encontramos estudios de caso como el del litigio comercial por el azúcar dentro del Mercosur en los que se describe una participación activa de los legisladores argentinos (Porcelli 2010; Schenoni y Astiria 2011). Estos casos corroboran la relevancia del efecto distributivo a la hora de entender la polarización partidaria en una votación de política externa.

Figura 3: Efectos marginales para el apoyo presidencial (Modelo 1)

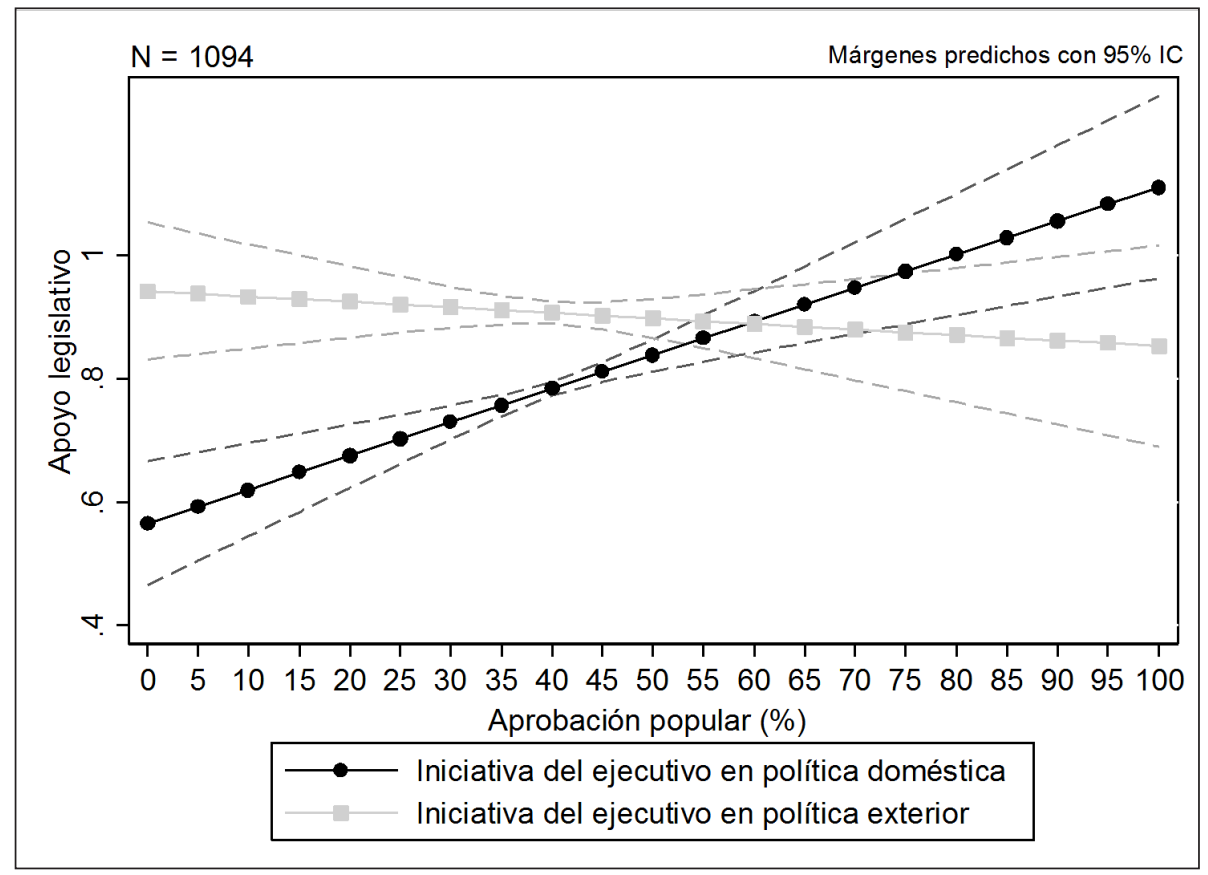


Las líneas punteadas son las referentes a las iniciativas presidenciales en el legislativo y vemos que las iniciativas de política externa poseen mayor apoyo legislativo hasta que las curvas se cruzan en 55\% de aprobación popular. A partir de ese nivel de aprobación popular, ambas dejan de diferenciarse al 95\% de confianza estadística. Es decir, que el modelo predice que las temáticas de política externa tendrán mayor o igual apoyo legislativo que las de política externa, independientemente del nivel de aprobación popular del que goce el Presidente.

Este resultado corrobora lo ya observado por Calvo (2007), quien muestra que - a pesar de los altos niveles de apoyo legislativo fruto de las negociaciones previas a las votaciones en el plenario- en Argentina, la agenda externa es prácticamente insensible a la aprobación popular del Presidente. Nótese, en este sentido, que mientras el apoyo de las iniciativas de política doméstica está positivamente asociado a la aprobación popular del Presidente, en materia exterior el efecto es casi nulo, apenas negativo.

La figura a continuación (ver Figura 4), refleja la prueba de hipótesis sobre el tamaño de la coalición de gobierno y su efecto en el apoyo legislativo $\left(H_{2}\right)$. Los resultados muestran que los cambios en el porcentaje del bloque legislativo que apoya al oficialismo no afectan de manera significativa las propuestas del Ejecutivo, tanto en política doméstica como externa. En el caso de las iniciativas del Legislativo, al contrario, el tamaño de la coalición afecta positivamente el nivel de apoyo conseguido en el plenario. Es importante destacar que, si bien estadísticamente significativa, la magnitud del efecto es pequeña. Estos resultados sobre $\mathrm{H}_{2}$ coinciden con los de Alemán y Calvo (2010), indicando que la ausencia de un gobierno unificado en Argentina no logra influir sobre el éxito legislativo del Presidente, inclusive cuando se trata de votaciones nominales. 
Figura 4: Efectos marginales para el apoyo presidencial (Modelo 1)

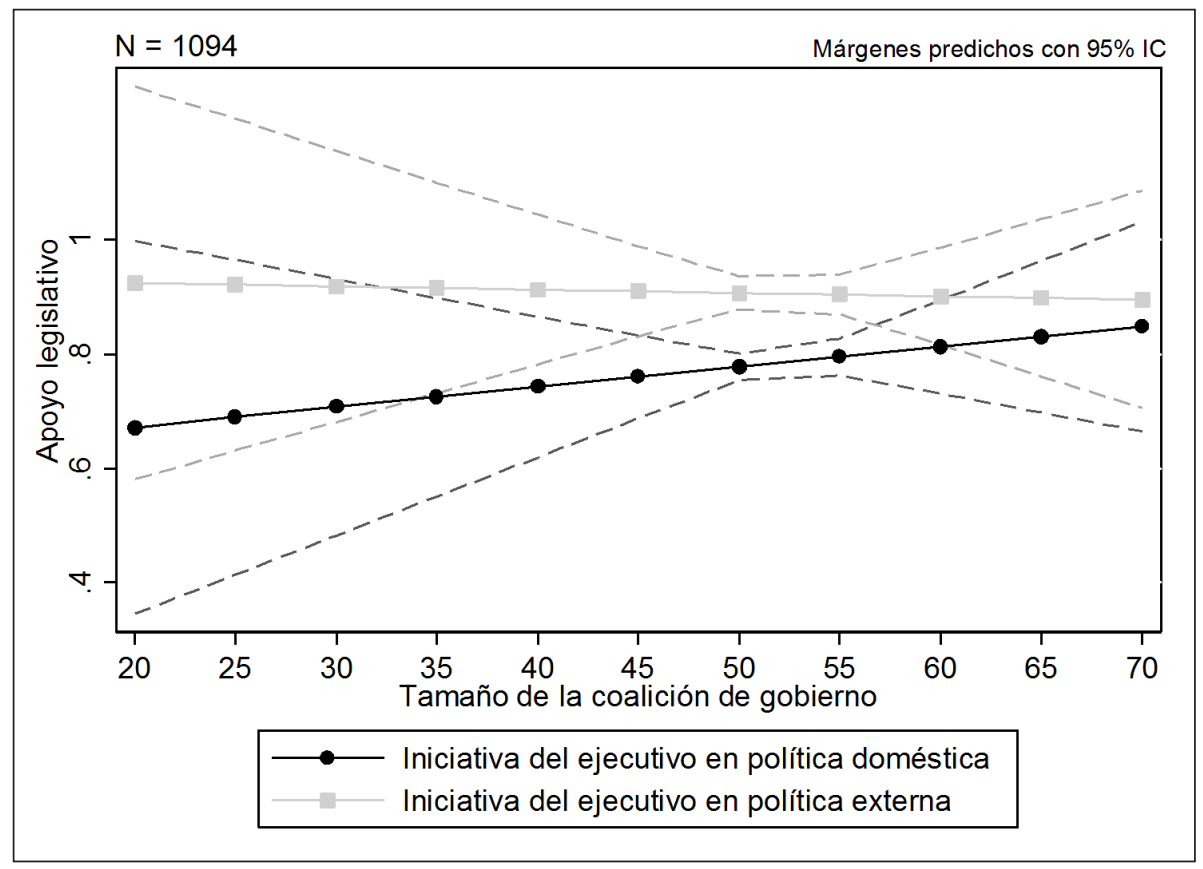

Finalmente, la Figura 5 corresponde a nuestra $H_{3}$. Observamos que la utilización de DNU posee un efecto negativo para ambas políticas, lo que prueba como válida nuestra hipótesis. Nuevamente, este resultado coincide con estudios previos basados en otros períodos históricos de la Argentina (Alemán y Calvo 2008), siendo este uno de los puntos de mayor conflictividad entre poderes Ejecutivo y Legislativo. 
Figura 5: Efectos marginales para el apoyo presidencial (Modelo 1)

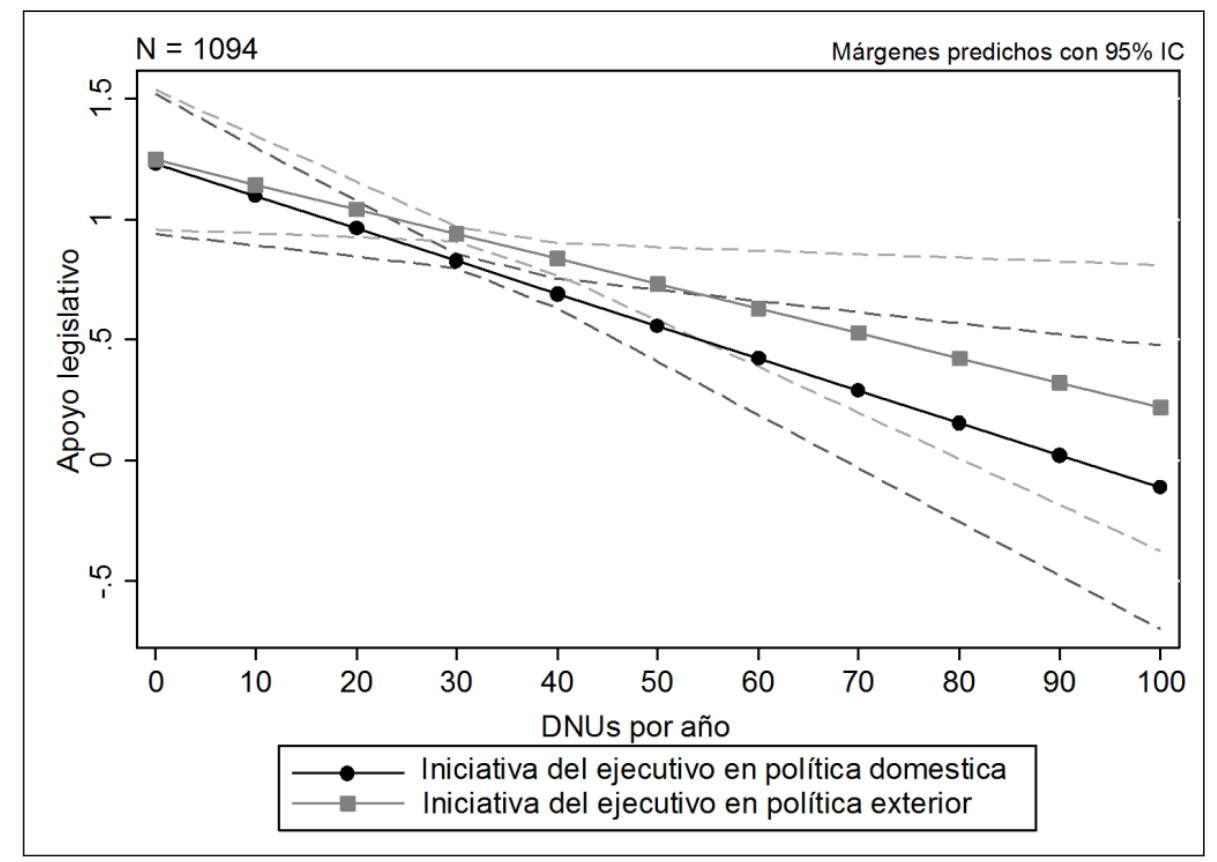

En lo que respecta a los controles, los resultados también son interesantes: muestran resultados consistentes y estadísticamente significativos para afirmar que durante el periodo estudiado (a) cuanto mejor la evaluación de la economía por la población, mayor el apoyo legislativo al Presidente, lo que es bastante intuitivo; (b) cuanto menor la polarización ideológica en el Congreso, mayor el apoyo legislativo al Presidente, lo que es coherente con la literatura; y (c) las iniciativas presentadas en el Senado poseen en promedio 10\% más apoyo legislativo que las que son propuestas en la Cámara.

\section{CONCLUSIÓN}

El presente trabajo hace dos contribuciones centrales a la literatura latinoamericana de estudios legislativos. La primera es la de probar la hipótesis de que el Presidente argentino obtiene mayor apoyo legislativo en las temáticas de política exterior cuando son comparadas a las temáticas de política doméstica. La tesis de los dos presidentes lo explica por el rol protagónico del Presidente como propulsor de la política exterior (first-mover) y por poseer una cantidad y calidad de informaciones en los asuntos internacionales mucho más grande que el Congreso. Esos factores institucionales incentivarían una delegación mayor del Congreso al Presidente en los asuntos exteriores, significando que rechazar estos acuerdos internacionales en el extranjero sea costoso para los legisladores. En el caso argentino, que es un presidencialismo multipartidista cuyo Presidente 
posee las ventajas institucionales mencionadas, nuestros resultados confirman la existencia de dos presidentes según el dominio temático de la política.

La segunda contribución se refiere a la comprensión del poder presidencial en general, más específicamente de la relación entre variables políticas e institucionales y del apoyo que el Presidente recibe en el Congreso Nacional. Como nuestra muestra de votaciones comprende los años 2001 a 2014, ofrecemos resultados empíricos actualizados para el caso argentino, cuyas principales investigaciones sobre la temática abarcaban los años ochenta hasta 2001 (Alemán y Calvo 2010).

Mientras que la popularidad presidencial afecta positivamente el nivel de apoyo legislativo a sus propuestas sobre política doméstica, ese efecto es nulo para la política externa. Esto demuestra un cierto grado de aislamiento de esta política. De manera general, el tamaño de la coalición de partidos que apoya al gobierno tampoco afecta el apoyo legislativo al Presidente, lo que corrobora la literatura especializada y dando fuerza a la teoría de que los presidencialismos multipartidarios no son tan inestables como anteriormente se imaginaba. Asimismo, corroboramos un efecto negativo del uso de DNU sobre el apoyo al Presidente y la relevancia de la polarización ideológica entre los partidos y el bicameralismo como factores que afectan de manera general el éxito legislativo de las iniciativas presidenciales.

\section{REFERENCIAS BIBLIOGRÁFICAS}

Alcántara, Manuel y Mercedes Garcia Montero. 2008. “Institutions and Politicians: An Analysis of the Factors that Determine Presidential Legislative Success". Working Paper recuperado en junio 2015 de https://kellogg.nd.edu/publications/workingpapers / WPS/348.pdf. Último acceso: junio de 2015.

Alemán, Eduardo y Ernesto Calvo. 2008. "Analyzing Legislative Success in Latin America: The Case of Argentina". En New Voices in the Study of Democracy, editado por Guillermo O'Donnell, Joseph Tulchin y Augusto Varas. Woodrow Wilson International Center for Scholars: Woodrow Wilson Center, 7-36.

Alemán, Eduardo y Ernesto Calvo. 2010. “Unified Government, Bill Approval, and the Legislative Weight of the President". Comparative Political Studies, 20(10): 1-24.

Alemán, Eduardo y Patricio Navia. 2009. "Institutions and Legislative Success of Strong Presidents: An analysis of Government Bills in Chile". The Journal of Legislative Studies 15 (4): 401-419.

Alemán, Eduardo y George Tsebelis. 2005. “The Origins of Presidential Conditional Agenda-Setting Power in Latin America". Latin American Research Review 40 (2): 3-26.

Altman, David. 2000. "Politics of Coalition Formation and Survival in Multiparty Presidential Democracies: Uruguay 1989-1999". Party Politics 6(3): 259-283.

Andersen, Robert. 2008. Modern Methods for Robust Regression. London, UK: Sage.

Anderson, Christopher J. y Matthew M. Singer. 2008. "The Sensitive Left and the Impervious Right: Multilevel Models and the Politics of Inequality, Ideology, and Legitimacy in Europe". Comparative Political Studies 20(10): 1-35. 
Badillo, Margarita Jiménez. 2007. “Gobernando sin mayorías parlamentarias en América Latina". Opinião Pública 13(1): 148-184.

Baldwin, Robert y Christopher Magee. 2000. Congressional Trade Votes: From NAFTA Approval to Fast-Track Defeat. Washington: Institute for International Economics.

Bertelli, Anthony, y Lilliard E. Richardson Jr. 2008. “Ideological Extremism and Electoral Design: Multimember versus Single Member Districts". Public Choice, 137(1-2): 347-368.

Bjereld, Ulf y Marie Demker. 2000. “Foreign Policy as Battle Field: A study of National Interest and Parties Motives". Scandinavian Political Studies 23(1): 17-36.

Bond, Jon y Richard Fleisher. 1990. The President in the Legislative Arena. Chicago: University of Chicago Press.

Burgin, Eileen. 1994. "Influences Shaping Members' Decision Making: Congressional Voting on the Persian Gulf War". Political Behavior 16(3): 319-342.

Calvo, Ernesto. 2007. "The Responsive Legislature: Public Opinion and Law Making in a Highly Disciplined Legislature". British Journal of Political Science 37(2): 263-280.

Calvo, Ernesto. 2013. "Representación política, política pública y estabilidad institucional en el Congreso argentino". En ¿Cuánto importan las instituciones? Gobierno, Estado y actores en la política argentina, editado por Carlos Acuña. Buenos Aires: Siglo XXI, 121-155.

Calvo, Ernesto e Iñaki Sagarzazu. 2011. "Legislator Success in Committee: Gatekeeping Authority and the Loss of Majority Control". American Journal of Political Science 55(1): $1-15$.

Canes-Wrone, Brandice, William Howell y David Lewis. 2008. "Toward a Broader Understanding of Presidential Power: A Reevaluation of the Two Presidencies Thesis". The Journal of Politics 70(1): 1-16.

Cárdenas, Mauricio; Roberto Junguito y Mónica Pachón. 2006. “Political Institutions and Policy Outcomes in Colombia: The Effects of the 1991 Constitution". Banco Interamericano de Desarrollo, Research Network Working Paper R-505.

Catterberg, Gabriela y Valeria Palanza. 2012. “Argentina: dispersión de la oposición y el auge de Cristina Fernández de Kirchner". Revista de Ciencia Política 32(1): 3-30.

Chasquetti, Daniel. 2001. "Democracia, multipartidismo y coaliciones en América Latina: evaluando la difícil combinación". En Tipos de presidencialismo y coaliciones políticas en América Latina, editado por Jorge Lanzaro. Buenos Aires: Consejo Latinoamericano de Ciencias Sociales/CLACSO, 319-359.

Cheibub, José Antonio, Adam Przeworski, y Sebastián M. Saiegh. 2004. "Government Coalitions and Legislative Success under Presidentialism and Parliamentarism". British Journal of Political Science 34(4): 565-87.

Conley, Richard. 1999. "Drealing Presidential Fast-Track Authority: The Impact of Constituency Pressures and Political Ideology on Trade Policy in Congress". Political Research Quarterly 52(4): 785-799.

Cox, Gary W. y Matthew McCubbins. 2001. "The Institutional Determinants of Economic Policy Outcomes". En Presidents, Parliaments, and Policy, edited by Stephan Haggard and Matthew McCubbins. Cambridge: Cambridge University Press, 21-63.

Dahl, Robert. 1950. Congress and Foreign Policy. New York: Norton.

Década Votada. (2001-2014). Buenos Aires, DF: Hacks/Hackers. Recuperado en mayo 2004 de http:/ / www.decadavotada.com.ar.

Edwards, George C. 1989. At the Margins: Presidential Leadership of Congress. New Haven: Yale University Press.

Eshbaugh-Soha, Matthew. 2010. "The Importance of Policy Scope to Presidential Success in Congress". Presidential Studies Quarterly 40(4): 708-724.

Feliú, Pedro y Janina Onuki. 2014. “Unidade partidária e política externa na América Latina”. Revista Brasileira de Ciências Sociais 29(86): 125-142.

Figueiredo, Argelina y Fernando Limongi. 1999. Executivo e Legislativo na nova ordem constitucional. Rio de Janeiro: FGV. 
Figueiredo, Argelina, Denise L. Salles y Marcelo M. Vieira. 2009. "Political and Institutional Determinants of Executive's Legislative Success in Latin America". Brazilian Political Science Review 3(2): 155-171.

Fleisher, Richard, Jon Bond, Glen Krutz y Stephen Hanna. 2000. “The Demise of the Two Presidencies". American Politics Quarterly 28(1): 3-25.

Fordham, Benjamin. 1998. "Economic Interests, Party and Ideology in Early Cold War Era U.S. Foreign Policy". International Organization 52(2): 359-396.

García Montero, Mercedes. 2009. Presidentes y Parlamentos ¿quién controla la actividad legislativa en América Latina? Madrid: Centro de Investigaciones Sociológicas.

Gelman, Andrew y Jennifer Hill. 2006. Data Analysis Using Regression and Multilevel/Hierarchical Models. Cambridge, UK: Cambridge University Press.

González, Luis Eduardo y Charles Guy Gillespie. 1994. "Presidentialism and Democratic Stability in Uruguay". En The Failure of Presidential Democracy: Comparative Perspectives (Vol. 2), edited by J. J. Linz and A. Valenzuela. Baltimore: Johns Hopkins University Press, 151-178.

Häge, Frank M. 2013. "Coalition Building and Consensus in the Council of the European Union". British Journal of Political Science 43(3): 481-504.

Henisz, Witold y Edward Mansfield. 2006. "Votes and Vetoes: The Political Determinants of Commercial Openness." International Studies Quarterly 50(1): 189-212.

Hiscox, Michael J. 2002. "Commerce, Coalitions, and Factor Mobility: Evidence from Congressional Votes on Trade Legislation." American Political Science Review 96(3): 593-608.

Holsti, Ole y James Rosenau. 1986. "Consensus Lost. Consensus Regained? Foreign Policy Beliefs of American Leaders, 1976-1980". International Studies Quarterly 30(4): 375-409.

Jones, Mark P., and Wonjae Hwang. 2005. "Party Government in Presidential Democracies: Extending Cartel Theory beyond the US Congress" American Journal of Political Science 49(2): 267-282.

Kasta, Veronica. 2009. “Liderazgo Político de Eduardo Duhalde: 'El hombre que nunca se rinde. El hombre que está condenado al éxito". Revista de Ciencia Política (Buenos Aires) 7(1). Recuperado en http:/ / www.revcienciapolitica.com.ar/num7art6.php.

King, Gary. 1986. "Political Parties and Foreign Policy: A Structuralist Approach". Political Psychology, 7(1): 83-101.

Kuenzi, Michelle, y Gina Lambright. 2005. "Party Systems and Democratic Consolidation in Africa's Electoral Regimes". Party Politics 11(4): 423-446.

Kupchan, Charles A. y Peter L. Trubowitz. 2007. “The Demise of Liberal Internationalism in the United States". International Security 32(2): 7-44.

Laakso, Markku y Rein Taagepera. 1979. "Effective number of Parties: A Measure with Application to West Europe." Comparative Political Studies 12(1): 3-27.

Lindsay, James y Randall Ripley. 1992. "Foreign and Defense Policy in Congress: A Research Agenda for the 1990s". Legislative Studies Quarterly 17(3): 417-449.

Mack, W.R., Karl DeRouen y David Lanoue. 2011. “Foreign Policy and Presidential Support in Congress". Foreign Policy Analysis 9(1): 79-102.

Mainwaring, Scott. 1993. "Presidentialism, Multipartism, and Democracy: The Difficult Combination". Comparative Political Studies 26 (2): 198-228.

Mainwaring, Scott. 1997. "Multipartism, Robust Federalism, and Presidentialism in Brazil", in Presidentialism and Democracy in Latin America, edited by Scott Mainwaring and Matthew Soberg Shugart. New York: Cambridge University Press, 55-109.

McCormick, James y Eugene Wittkopf. 1990. "Bipartisanship, Partisanship and Ideology in Congressional: Executive Foreign Policy Relations, 1947-1988". The Journal of Politics 52(4): 1077-1100.

McCormick, James y Eugene R. 1992. Wittkopf. “At the Water's Edge. The Effects of Party, Ideology, and Issues on Congressional Foreign Policy Voting, 1947 to 1988". American Politics Quarterly 20(1): 26-53.

McCormick, James, Eugene Wittkopf y David Danna. 1997. "Politics and Bipartisanship at the Water's Edge: A Note on Bush and Clinton". Polity 30(1): 132-49. 
McCoy, Terry L. 1971. "Congress, the President, and Political Instability in Peru" En Latin American Legislatures: Their Role and Influence, Analyses for Nine Countries, edited by W. H. Agor. New York: Praeger, 325-366.

Meernik, James. 1993. "Presidential Support in Congress: Conflict and Consensus on Foreign and Defense Policy". The Journal of Politics 55(3): 569-587.

Milner, Helen V. y Dustin Tingley. 2012. Sailing the Water's Edge: Where Domestic Politics meets Foreign Policy. Working Paper recuperado en febrero 2015 de http: / /irworkshop.sites. yale.edu/sites/default/files/milnertingleywatersedge.pdf.

Molinas, José; Aníbal Pérez-Liñan y Sebastián Saiegh. 2004. "Political Institutions, Policymaking Processes, and Policy Outcomes in Paraguay, 1954-2003". Revista de Ciencia Política 24(2): 67-93.

Molinelli, Guillermo; Valéria Palanza y Gisela Sin. 1999. Congreso, Presidencia y Justicia en Argentina. Buenos Aires: Eudeba.

Negretto, Grabiel. 2006. "Minority Presidents and Democratic Performance in Latin America". Latin American Politics and Society 48(3): 63-92.

Pereira, Carlos y Marcus A. Melo. 2012. "The Surprising Success of Multiparty Presidentialism". Journal of Democracy 23(3): 156-170.

Porcelli, Emanuel. 2010. "Parlamento y política exterior: algunas reflexiones en torno a la 'Ley del Azúcar'”. Meritum 5(2): 349-375.

Prins, Brandon C. y Bryan B. Marshall. 2001. "Congressional Support of the President: A Comparison of Foreign, Defense, and Domestic Policy Decision Making during and after the Cold War". Presidential Studies Quarterly 31 (4): 660-678.

Radcliff, Benjamin. 2001. "Organized Labor and Electoral Participation in American National Elections". Journal of Labor Research 22(2): 405-414.

Ripley, Randall and James Lindsay (eds.). 1993. Congress Resurgent: Foreign and Defense Policy on Capitol Hill. Ann Arbor: The University of Michigan Press.

Rousseeuw, Peter J., y Annick M. Leroy. 2005. Robust Regression and Outlier Detection. New York: Wiley.

Rudalevige, Andrew. 2002. Managing the President's Program: Presidential Leadership and Legislative Policy Formulation. Princeton: Princeton University Press.

Sanchez, Leandro Enrique. 2014. "Éxito y participación legislativa en materia de política exterior. El Congreso de la Nación Argentina, un estudio de caso". Revista Mexicana de Análisis Político y Administración Pública 3(2): 71-92.

Sanchez, Leandro Enrique. 2015. "El papel del Congreso Nacional de Argentina en la elaboración de la política exterior (1999-2003). Un modelo para su análisis”. Colombia Internacional 83: 103-132.

Santos, Manoel Leonardo; Aníbal Perez-linan y Mercedes Garcia Montero. 2014. “El control presidencial de la agenda legislativa en América Latina". Revista de Ciencia Política 34(3): 511-536.

Santos, Wanderley Guilherme dos. 1986. Sessenta e quatro: anatomia da crise. São Paulo: Vértice.

Schenoni, Luis L. y Alejo M. F. Aztiria. 2011. “El Congreso en la formulación de política exterior: Argentina y Brasil en perspectiva comparada". Densidades (8): 79-101.

Schraufnagel, Scott, y Stephen Shellman. 2001. "The Two Presidencies, 1984-98: A Replication and Extension". Presidential Studies Quarterly (31): 699-707.

Shugart, Matthew Soberg y John M. Carey. 1992. Presidents and Assemblies: Constitutional Design and Electoral Dynamics. Cambridge: Cambridge University Press.

Sinclair, Barbara. 1993. "Congressional Party Leaders in the Foreign and Defense Policy Arena". En Congress Resurgent: Foreign and Defense Policy on Capitol Hill, editado por Randall Ripley y James Lindsay. Ann Arbor: University of Michigan Press, 207-231.

Stepan, Alfred y Cindy Skach. 1993. "Constitutional Frameworks and Democratic Consolidation: Parliamentarianism versus Presidentialism". World Politics (46): 1-22.

Teyssier, Ronan. 2011. "The Organizational and Electoral Determinants of the Provincial Funding of Private Education in Canada: A Quantile Regression Analysis". Canadian Journal of Political Science 44(4): 829-857. 
White, Halbert. 1980. "A Heteroskedasticity-Consistent Covariance Matrix Estimator and a Direct Test for Heteroskedasticity". Econometrica 48(4): 817-838.

Wildavsky, Aaron. 1966. "The Two Presidencies". Society 4(2): 7-14.

Xie, Tao. 2004. "Congressional Roll Call Voting on China Trade Policy". American Politics Research 32(6): 679-697.

Pedro Feliú Ribeiro es profesor de Relaciones Internacionales en la Universidad de São Paulo (IRI-USP), investigador del Centro de Estudios de Negociaciones Internacionales (CAENI-USP) y Doctor en Ciencia Política por la Universidad de São Paulo. Actualmente es vice-presidente del programa de posgrado de IRI-USP. Correo electrónico: pedrofeliu@usp.br

Francisco Urdinez Doctor en Relaciones Internacionales por la Universidad de São Paulo y King's College de Londres (doble diploma). Profesor asistente en el Instituto de Ciencia Política de la Pontificia Universidad Católica de Chile. Correo electrónico: furdinez@uc.cl 
- 